\title{
¿Cuál debería ser el perfil de quien se denomine investigador en Colombia y Perú?
} How should be the profile of who called as researcher in Colombia and Peru?

\author{
Dice: Vicente A. Benites-Zapata4 ,
} Debe decir: Dice: Vicente A. Benites-Zapata5,

Correspondencia Alfonso J. Rodriguez-Morales arodriguezm@utp.edu.co

Recibido: 21/09/2016 Aprobado: 28/0g/2016

Citar como: Rodriguez-Morales AJ, Díaz-Vélez C, GálvezOlórteguiT, Benites-Zapata VA, Gálvez-Olórtegui I. ¿Cuál debería ser el perfil de quien se denomine investigador en Colombia y Perú?. Acta Med Peru. 2016;33(3):256-8

\author{
Alfonso J. Rodriguez-Morales', Cristian Díaz-Vélez 2,3, Tomás Gálvez- \\ Olórtegui ${ }^{4}$, José Gálvez-Olórteguii ${ }^{4}$ Vicente A. Benites-Zapata ${ }^{5}$ \\ 1 Grupo de Investigación Salud Pública e Infección, Facultad de Ciencias de la Salud, Universidad Tecnológica de Pereira. \\ Pereira, Colombia. \\ 2 Facultad de Medicina, Universidad San Martín de Porres, Chiclayo, Perú. \\ 3 Comité de Investigación, Hospital Nacional Almanzor Aguinaga Asenjo, EsSalud. Chiclayo, Perú. \\ 4 Scientia Clinical and Epidemiological Research Institute. Trujillo, Perú. \\ 5 Centro de Investigación de Salud Pública, Instituto de Investigación, Facultad de Medicina Humana, Universidad de San \\ Martín de Porres. Lima, Perú.
}

\section{Sr. Editor:}

La actividad de investigación científica es considerada a nivel mundial un trabajo formal y a la vez responsable, que puede afectar positiva o negativamente la situación en cada país ${ }^{[1,2]}$. Por ello, las universidades deben ser coherentes con respecto a la formación y el desarrollo de competencias en investigación, a través de actividades curriculares y extracurriculares que permitan el desarrollo de la actividad científica. Además, es deber de estas instituciones y la comunidad científica estar vigilantes y ser garantes del uso apropiado de los títulos académicos, y el calificativo de docentes e investigadores ${ }^{[3,4]}$.

La comunidad científica internacional reconoce que el calificativo de investigador se refleja a través de evidencia verificable, artículos en publicaciones científicas disponibles en revistas indizadas en bases de datos internacionales (ISI/WoK, Medline/PubMed, Scopus). En el caso específico de la medicina, es como si un "cirujano" sin record quirúrgico, sin cirugías, ni operaciones se autodenomine cirujano y quiera enseñar cómo hacerlo. Esto equivale a, autodenominarse investigador sin tener publicaciones, pudiendo ser solo un teórico de la investigación, que no ha demostrado competencia a la hora de concluir un proyecto de investigación que termine con la publicación de un artículo o en algunos casos con patente, donde la difusión de nuevo conocimiento beneficia no solo a la comunidad médica sino al paciente. Dicho de otra forma, no debe llamarse investigador a quien nunca ha investigado y comunicado sus resultados en una publicación científica verificable en alguna base de datos internacional más allá del grado académico alcanzado (maestría o doctorado) ${ }^{[4]}$.

A nivel mundial la formación académica en investigación es un desafío, y completar los niveles de maestría y doctorado puede tomar entre 5 y 7 años dependiendo de cada programa; periodos que se acompañan de un alto nivel de aprendizaje, teorización y especialización, finalmente plasmado en una tesis publicada en una revista indizada. Un profesional con grado de doctor (PhD) con 5 años de graduado y ninguna publicación, es como un piloto que jamás ha volado, siendo en este caso el título nada más que un ornamento. Latinoamérica, al igual que los países desarrollados, establece que un graduado de un programa de maestría/doctorado debe crear tecnología y participar activamente de la generación de conocimiento científico; es decir artículos derivados directa o indirectamente de su investigación.

Países como Colombia y Perú, a través de Colciencias (Departamento Administrativo Nacional de Ciencia y Tecnología de Colombia) y CONCYTEC (Consejo Nacional de Ciencia, Tecnología e Innovación 
Tabla 1. Comparación del número de publicaciones, revistas y número de investigadores reconocidos en Perú por Concytec y en Colombia por Colciencias.

\begin{tabular}{|c|c|c|c|c|}
\hline & \multicolumn{2}{|c|}{ Colombia } & \multicolumn{2}{|c|}{ Perú } \\
\hline & $\mathbf{N}$ & $(\%)$ & $\mathbf{N}$ & $(\%)$ \\
\hline \multicolumn{5}{|l|}{ Publicaciones Scopus 2015* } \\
\hline Total & 7500 & $(100)$ & 1813 & (100) \\
\hline Medicina & 2013 & $(26,8)$ & 798 & $(44,0)$ \\
\hline \multicolumn{5}{|l|}{ Revistas indizadas Scopus $2015^{*}$} \\
\hline Total & 78 & $(100)$ & 7 & $(100)$ \\
\hline Medicina & 14 & $(18,0)$ & 2 & $(28,2)$ \\
\hline \multicolumn{5}{|l|}{ Personas } \\
\hline Registradas & 66028 & (100) & 62018 & (100) \\
\hline Reconocidos como investigador $† \ddagger$ & 10042 & $(15,2)$ & 1269 & $(2,1)$ \\
\hline Senior & 1218 & $(12,1)$ & na & \\
\hline Asociado & 2767 & $(27,6)$ & na & \\
\hline Junior & 6057 & $(60,3)$ & na & \\
\hline
\end{tabular}

* Tomado de ranking scimago de países y revistas: www.scimajojr.com. Acceso: 21/09/16.

† Para Colombia: resultados de Colciencias 2015-2016 de investigadores (http://colciencias.gov.co/sites/default/files/listado-publicacion-resultadosfinales-conv737investigadores-consulta.pdf). Acceso: 21/09/16.

‡ Para Perú: Directorio Nacional de Investigadores: https://dina.concytec.gob.pe/appDirectorioCTI/. Acceso: 21/09/16.

na: en Perú aun no estratifican a los investigadores por niveles.

Tecnológica de Perú) respectivamente, han definido bajo criterios específicos, quién es y quién no es, un investigador; registros que se encuentran disponibles a través de sistemas de hojas de vida (CvLAC para Colombia, http://www.colciencias.gov. co/sites/default/files/upload/convocatoria/resumenEjecutivo. pdf, y el Directorio Nacional de Investigadores (DINA), http:// dina.concytec.gob.pe/appDirectorioCTI/, para Perú). Colciencias ha definido como investigador junior a una persona que se haya graduado con formación de doctorado, que sea integrante de un grupo de investigación y que haga parte de un proyecto de investigación del grupo o que se haya graduado de doctorado, o de maestría, o de alguna especialidad clínica con 1 producto de nuevo conocimiento o de resultados de actividades de desarrollo tecnológico e innovación tipo $A$, en toda su trayectoria y 4 productos de nuevo conocimiento o de resultados de actividades de desarrollo tecnológico e innovación, en los últimos 5 años. Del mismo modo que CONCYTEC los define como "personas naturales que poseen capacidades establecidas de acuerdo a una calificación, para realizar labores de investigación científica y/o desarrollo tecnológico"; basados en el grado académico, publicación de un libro, artículos publicados en revistas indizadas en bases de datos internacionales, patentes, registro de autor, formación de recursos humanos y presentación en congresos nacionales e internacionales (https://sites.google.com/a/ concytec.gob.pe/manual-dina/regina).

Este tipo de discusiones cobran mayor relevancia, cuando una universidad contrata a docentes sin publicaciones para dictar clases de investigación [5], o cursos y talleres de educación continua; sin contar con experiencia verificable, sin publicaciones, ni con el reconocimiento de las agencias de ciencia del país
(Colombia o Perú). Esta realidad se ha discutido recientemente en torno a cursos de investigación en universidades del norte de Perú, donde los instructores, en su gran mayoría son personas sin publicaciones en revistas indizadas de importancia (ISI/ WoK, Medline/PubMed, Scopus), ni son reconocidos como investigadores por el CONCYTEC. Lo que genera una visión errónea de la investigación en los participantes a dichos eventos, aun contando con investigadores calificados (Tabla 1).

Lamentablemente, en América Latina, los criterios con los cuales se eligen conferencistas no siempre obedecen a un entendimiento cabal del perfil de quien se denomine investigador. Tristemente, en la mayoría de casos, esta elección se sustenta en el amiguismo en lugar de la experiencia real o producción científica; siendo claramente responsabilidad de las autoridades universitarias como decanos o vicerrectores de investigación, que desafortunadamente como se ha demostrado $[6,7]$, pueden desconocer completamente la importancia del tema. En tal caso una opción factible, sería la necesidad de contar con publicaciones en revistas indizadas para la promoción a docente asociado o principal en las facultades de medicina, como se realiza en otros países en vías de desarrollo ${ }^{[8]}$. De esta forma no sólo se logrará una práctica médica basada en evidencias, sino una formación estudiantil y enseñanza basada en evidencias, verificable a través de las investigaciones publicadas por los docentes ${ }^{[9]}$.

La comunidad científica debe tener una actitud crítica y vigilante, decir no a la charlatanería, a la informalidad, desenmascarar a los farsantes y velar por una actividad científica seria y formal que conduzca al progreso científico. Por tal motivo, las universidades 
deben prohibir que personas sin calificación de CONCYTEC en Perú o Colciencias en Colombia, se autodenominen "Investigadores", que sean asesores de proyectos o incluso jurado de tesis; pues este reconocimiento se obtiene, de forma académica, y con formación y producción científica, ambas necesarias. Más aún los profesionales deben tomar conciencia y declinar invitaciones a dictar cursos o asignaturas de áreas temáticas en las que no puedan demostrar experiencia real, o publicaciones científicas.

Es propicia la ocasión para solicitar a instituciones como la SUNEDU (Superintendencia Nacional de Educación Superior Universitaria) y el SINEACE (Sistema Nacional de Evaluación, Acreditación y Certificación de la Calidad Educativa) en Perú, o el CESU (Consejo Nacional de Educación Superior) y el CNA (Consejo Nacional de Acreditación) en Colombia, que como parte de la acreditación de las universidades se solicite que los docentes que enseñan los cursos de investigación y de tesis sean investigadores acreditados por CONCYTEC en Perú o Colciencias en Colombia, respectivamente.

El objetivo final de toda actividad científica va más allá de la generación de conocimiento científico y es la de formar nuevos y mejores investigadores, tan necesarios en países como Perú y Colombia.

Fuente de financiamiento: Los autores declaran no haber recibido ninguna financiación para la realización de este trabajo.

Conflicto de intereses: $A J R M$ es reconocido como investigador Senior por Colciencias, CDV, TGO, VABZ y JGO son reconocidos como investigadores en el REGINA de Concytec.

\section{REFERENCIAS BIBLIOGRÁFICAS}

1. Paniz-Mondolfi $A E$, Rodríguez-Morales AJ. Venezuelan science in dire straits. Science. 2014;346(6209):559.

2. Rodríguez-Morales AJ, Mayta-Tristán P. Challenges of Colombian medical journals. Lancet. 2014;384(9953):1503-4.

3. Alfaro-Toloza P, Olmos-de-Aguilera R, Rodríguez-Morales AJ. Latin American Undergraduate Medical Journals. Med Educ Online. 2014;19. doi: 10.3402/meo.v19.25901.

4. Taype-Rondán A, Peña-Oscuvilca A, Rodríguez-Morales AJ. Producción científica de los docentes de cursos de investigación en facultades de medicina de Latinoamérica: ¿se está dando ejemplo? FEM. 2013;16(1):5-6

5. Pereyra-Elías R, Huaccho-Rojas JJ, Taype-Rondan Á, Mejia CR, Mayta-Tristán P. Publicación y factores asociados en docentes universitarios de investigación científica de escuelas de medicina del Perú. Rev Peru Med Exp Salud Publica. 2014;31(3):424-30.

6. Culquichicón-Sánchez CG, Gil-Restrepo AF, Rodríguez-Morales AJ. Baja producción científica de decanos en facultades de medicina y salud de Colombia: ¿una realidad común en Latinoamérica? Salud Publica Mex. 2016;58(4):402-3.

7. Urquia-Osorio $\mathrm{H}$, Henriquez-Marquez KI, Vásquez-Bonilla WO, Estrada-Mendoza AJ, Rodríguez-Morales AJ. Producción científica de decanos de medicina y salud de universidades centroamericanas. Salud Pública de Méx. 2014;56(3):243-4.

8. Sukhlecha A. Research publications: Should they be mandatory for promotions of medical teachers? J Pharmacol Pharmacother. 2011;2(4):221-4.

9. Petty G. Evidence-based teaching a practical approach. 2nd Edition. Oxford: Oxford University Press; 2014.

\title{
Las ediciones anteriores de Acta Médica Peruana están disponibles en:
}

\author{
www.redalyc.org
}

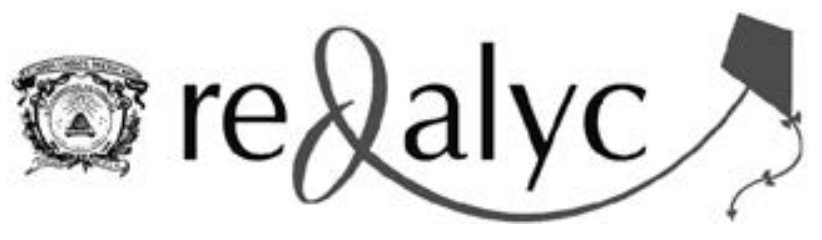

